\title{
Line Profile Variability in the Be Star $\alpha$ Eridani
}

\author{
N. V. Leister and E. Janot-Pacheco \\ Instituto Astronômico e Geofísico, Universidade de São Paulo, Brazil, \\ 04301-974
}

\section{J. Leyton Z.}

Departamento de Física, Universid de la Serena, Chile

\author{
A. M. Hubert and M. Floquet \\ Observatoire de Paris, Section d'Astrophysique de Meudon, UMR 8633 \\ CNRS/DASGAL, F-92195 Meudon Cedex, France
}

\begin{abstract}
We present an analysis of line profile variability of the Be star $\alpha$ Eri (Achernar). Spectra of He I $\lambda 667.8 \mathrm{~nm}$ were collected at Brazilian Laboratório Nacional de Astrofísica (LNA) during 10 observing runs covering 27 nights in 6 years. The presence of a 1.26 days period reported previously is confirmed and we report also the detection of other periods ( 0.73 and 0.53 days) and a possible 12.5 days modulation. A transient high-velocity absorption component with $R V=-500 \mathrm{~km} / \mathrm{s}$, higher than $-v \sin i(-220 \mathrm{~km} / \mathrm{s})$ has appeared at the end of 1993 , near the presumed maximum of the $\mathrm{H} \alpha$ emission phase.
\end{abstract}

\section{Introduction}

The brightest Be star $\alpha$ Eri (Achernar, HD 10144, B3-4Ve, $v \sin i=220 \mathrm{~km} / \mathrm{s}$ ) has been reported to have a strong variability in $\mathrm{H} \alpha$ going through a $\mathrm{B}$ to $\mathrm{Be}$ phase and vice-versa. From intensive photometry in 1985-86 and medium-dispersion spectroscopy obtained simultaneously, Balona et al. (1987) concluded to a 1.26 $\mathrm{d}$ period in both radial velocity and light variations, however the light curve was described as complex and varying with time. This star has been included in the intensive spectroscopic survey of bright Be stars conducted at LNA.

\section{Spectroscopic observations}

The He I $\lambda 667.8 \mathrm{~nm}$ line has been monitored over one or two seasons each year (except 1994) since 1992. 1368 spectra $(S / N$ about 300$)$ were obtained with $R=50000$ and $0.008 \mathrm{~nm} /$ pixel, see Table 1 . This line is seen as a broad variable absorption with sometimes outer $V$ and/or $R$ emission components highly variable. The last observations made on 1998 November 30 and December 01 re- 


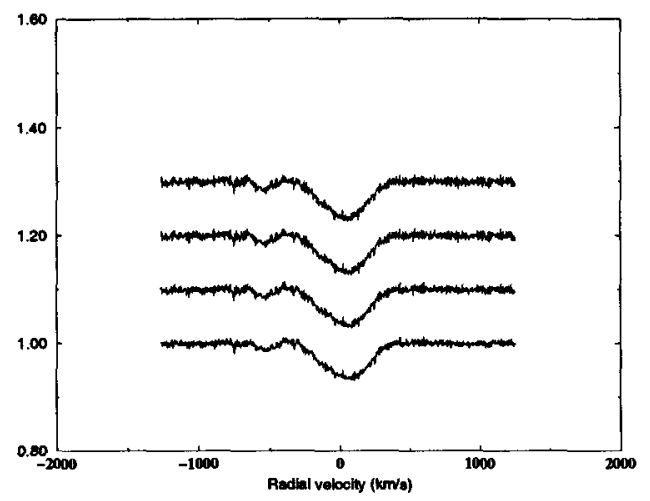

Figure 1. He I $\lambda 667.8 \mathrm{~nm}$ for 1993 .

vealed a gradual increase of both emissions over two days. The peak separation is estimated to be $600 \mathrm{~km} / \mathrm{s}$.

Table 1. Log of spectroscopic observations at $L N A$.

\begin{tabular}{crc} 
Date & \multicolumn{1}{c}{ MHJD $^{a}$} & Number of Spectra \\
\hline 1992 & $636.428-905.737$ & 283 \\
1993 & $1227.471-1263.617$ & 274 \\
1995 & $1969.718-2054.534$ & 309 \\
1996 & $2411.563-2416.739$ & 317 \\
1997 & $2764.706-2766.833$ & 80 \\
1998 & $3148.584-3419.667$ & 105 \\
\hline
\end{tabular}

In 1992 the line profile is sometimes disturbed by blue or red spikes located at about $\pm 250 \mathrm{~km} / \mathrm{s}$. They are generally observed on alternate sides of the line.

At the end of 1993 a high-velocity absorption component appeared on the blue edge of the photospheric line with $R V=-500 \mathrm{~km} / \mathrm{s} \mathrm{(Fig.} \mathrm{1)} \mathrm{much} \mathrm{more}$ negative than $-v \sin i$. It was observed over three days, of our run, giving evidence of stellar activity. Such a transient high-velocity satellite absorption has been detected in another Be star, $\mu$ Cen (Rivinius et al. 1998) during a precursor phase as described by these authors. Some $\mathrm{H} \alpha$ profiles obtained in 1993, 1994, 1995 and 1998 have revealed that $\alpha$ Eri has started a Be phase from the beginning of nineties. Indeed, emission is already present at beginning of 1992 according to data contained in the atlas published by Hanuschik et al. 1996. It seems to go through a maximum in 1993-94; afterwards it decreased and in 1995 it appeared as a broad photospheric line with both emission peaks above the continuum level. At the end of 1998 it has turned to a complex absorption profile with a deep core superimposed to the photospheric line, giving an aspect of a B-shell type phase. Probably very weak $\mathrm{V}$ and $\mathrm{R}$ emissions disturbed also this composite profile. Nevertheless at the same time the He I $\lambda 667.8 \mathrm{~nm}$ has shown a rapid 


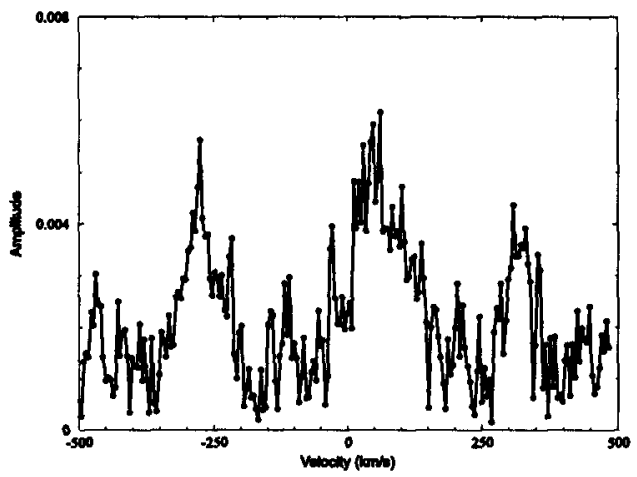

Figure 2. Amplitude distribution $(\mathrm{f}=1.36 \mathrm{c} / \mathrm{d}, l p v)$.

increase in $V$ and $R$ emission peaks over two days, indicating the start of a new activity phase.

\section{Periodic variations}

The search for periodicity in $\alpha$ Eri was performed through Fourier analysis enhanced by methods that try to eliminate aliases due to data sampling. They are the CLEAN (Roberts et al. 1987) and the CLEANEST (Foster 1995) algorithms. These methods were applied to the radial velocity $(R V)$ of the centroid, the full width at half maximum ( $F W H M$ ) and to the time series formed by data at each resolution step across the He I $\lambda 667.8 \mathrm{~nm}$ line profile (lpv).

Photometric data obtained over 1985 and 1986 seasons and published by Balona et al. (1987) were re-analyzed with the same methods. Hipparcos photometry (from mid-1989 to mid-1993) was also investigated.

Results are given in Table 2. Frequencies are given by order of decreasing amplitude. The most conspicuous one is the frequency $1.36 \mathrm{c} / \mathrm{d}$ present both in spectroscopic and photometric data (Fig. 2). Its first harmonic is also present in spectroscopic data. The associated signal is detected a wavelength range

Table 2. Main frequencies found.

\begin{tabular}{ccccccc} 
Frequencies & FWHM $^{a}$ & $R V^{a}$ & Line $^{a}$ & Line $^{b}$ & Photometry $^{a}$ & Hipparcos $^{a}$ \\
\hline 1.36 & \multirow{2}{*}{} & & $\mathrm{X}$ & $\mathrm{X}$ & $\mathrm{X}$ & \\
1.90 & $\mathrm{X}$ & $\mathrm{X}$ & $\mathrm{X}$ & $\mathrm{X}$ & $\mathrm{X}$ & \\
0.79 & & & $\mathrm{X}$ & $\mathrm{X}$ & $\mathrm{X}$ & $\mathrm{X}$ \\
2.60 & & & $\mathrm{X}$ & $\mathrm{X}$ & & \\
0.08 & & & $\mathrm{X}$ & $\mathrm{X}$ & $\mathrm{X}$ & \\
\hline
\end{tabular}

${ }^{\text {aCLEAN method }}$

${ }^{b}$ CLEANEST algorithm

$(650 \pm 50 \mathrm{~km} / \mathrm{s})$ higher than $2 v \sin i$. Furthermore it can be noted that even 


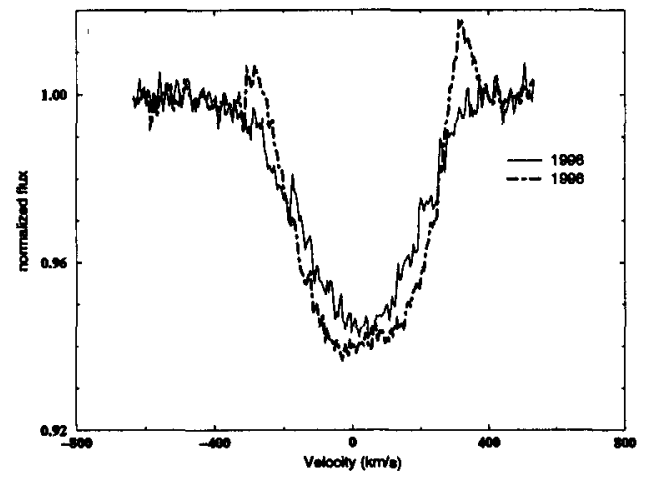

Figure 3. The mean line profile He I $\lambda 667.8 \mathrm{~nm}-1996-1998$.

when $V$ and $R$ emissions are missing the total width of the He I $\lambda 667.8 \mathrm{~nm}$ is larger then $2 v \sin i$, see Fig. 3 . The higher frequency $1.90 \mathrm{c} / \mathrm{d}$ is only detected in spectroscopic data.

The $0.79 \mathrm{c} / \mathrm{d}$ previously detected by Balona et al. (1987) is found in lpv and photometric data. It is probably associated with the stellar rotation period. From velocity phases across the line profile we could obtain an estimation of $g$-modes characteristics, see in Table 3 .

Table 3. $\quad l,|m|$ determinations.

\begin{tabular}{cr} 
Frequencies & $l,|m|$ \\
\hline $1.36(2.60)$ & $2-3,2-3$ \\
1.90 & $2-3$
\end{tabular}

In conclusion, several frequencies have been confirmed or detected in $\alpha$ Eri and possibly a beat modulation. A transient high-velocity absorption component much more negative than $-v \sin i$ has been observed end 1993, near a presumed maximum of the Be phase. At the end of 1998, a new period of activity is conspicuous in the He I $\lambda 667.8 \mathrm{~nm}$ as $\mathrm{H} \alpha$ is seen as a complex absorption.

\section{References}

Balona, L.A., Engelbrecht, C.A., Marang, F. 1982, MNRAS 227, 123

Foster, G. 1995, AJ 109, 1889

Hanuschik, R.W., Hummel, W., Sutorius, F., Dietle, O., Thimm, G. 1996, A\&AS 116,309

Rivinius, Th., Baade, D., Stefl, S. Stahl, O., Wolf, B., Kaufer, A. 1998, A\&A 333,125

Roberts, D.H., Lehár, J., Dreher, W. 1987, AJ 93, 968 